\title{
Almost Sure Limit Theorems for Multivariate General Standard Normal Sequences and Applications
}

\author{
Zhicheng Chen ${ }^{1,2}$ and Xinsheng Liu $\mathbb{D}^{1}$ \\ ${ }^{1}$ State Key Laboratory of Mechanics and Control of Mechanical Structures, \\ Key Laboratory for Intelligent Nano Materials and Devices of the Ministry of Education, \\ Institute of Nano Science and Department of Mathematics, Nanjing University of Aeronautics and Astronautics, \\ Nanjing 210016, China \\ ${ }^{2}$ Department of Mathematics, Henan Institute of Science and Technology, Xinxiang 453003, China
}

Correspondence should be addressed to Xinsheng Liu; xsliu@nuaa.edu.cn

Received 9 December 2019; Revised 17 March 2020; Accepted 23 March 2020; Published 18 May 2020

Academic Editor: Chaudry M. Khalique

Copyright (C) 2020 Zhicheng Chen and Xinsheng Liu. This is an open access article distributed under the Creative Commons Attribution License, which permits unrestricted use, distribution, and reproduction in any medium, provided the original work is properly cited.

Under suitable conditions, the almost sure central limit theorems for the maximum of general standard normal sequences of random vectors are proved. The simulation of the almost sure convergence for the maximum is firstly performed, which helps to visually understand the theorems by applying to two new examples.

\section{Introduction}

The classical extreme value theory studies extreme phenomena in nature and human society, such as floods, hurricane, stock market crash, megaseism, and so on [1-3]. Almost sure (a.s.) convergence is a nice behavior of the various ways of convergence $[4,5]$. The almost sure central limit theorem (ASCLT) on partial sums is initiated by Brosamler and Schatte for independent identically distributed (i.i.d.) random variables $[6,7]$. Let $X_{1}, X_{2}, \ldots$ be i.i.d. random variables with $E\left(X_{n}\right)=0, \operatorname{Var}\left(X_{n}\right)=1$ and $T_{n}=$ $\sum_{k=1}^{n} X_{k}$. Under some regularity conditions, we have

$$
\lim _{n \longrightarrow \infty} \frac{1}{\log n} \sum_{k=1}^{n} \frac{1}{k} I\left(\frac{T_{k}}{\sqrt{k}} \leq x\right)=\Phi(x), \quad \text { a.s., }
$$

for any $x$, where $I, \log n$, and $\Phi(x)$ denote the indicator function, the natural logarithm, and the standard normal distribution function. Later, Lacey and Philipp prove that equation (1) holds when $f$ is a bound Lipschitz function [8]. The almost sure central limit theorem on maximum of i.i.d random variables is firstly discovered by Fahrnar and Stadtmüller and Cheng et al., respectively [5,9]. Berkes et al. consider the general nonlinear functionals case [10]. Csáki and Gondigdanzan investigate ASCLT for the maximum of a stationary Gaussian sequences [11]. Chen and Lin extend to the nonstationary Gaussian sequences [12]. Chen and Peng provide an ASCLT for the maxima of multivariate stationary Gaussian sequences under some mild conditions $[13,14]$.

In recent years, the almost sure convergence of the joint distribution is explored. The almost sure limit theorems related to the maxima of the complete and incomplete samples for stationary sequences are considered [15]. Peng et al. extend the result to the maxima and minima of the complete and incomplete samples for weakly dependent stationary Gaussian sequences [16]. The ASCLT for the maxima and sums of i.i.d. random variables is investigated by Zang et al. [17]. Tan and Wang consider ASCLT for the maxima and sums of standardized stationary Gaussian sequences under some conditions [18]. The almost sure convergence of the maxima and minima for stationary sequences holds under some dependence conditions [19]. The behavior of almost sure convergence of the maxima and the minima for a strongly dependent stationary Gaussian sequence is extended to multivariate vectors $[20,21]$.

In this paper, we study ASCLT of the multivariate general standard normal sequences under some suitable conditions. Throughout this paper, $\left\{X_{1}, X_{2}, \ldots\right\}$ is a standardized normal sequence of $d$-dimensional random vector, i.e., each 
component of the random vector has a zero mean and a unit standard deviation. Set

$$
\begin{aligned}
r_{i j}(p) & =\operatorname{Cov}\left(X_{i}(p), X_{j}(p)\right), \\
r_{i j}(p, q) & =\operatorname{Cov}\left(X_{i}(p), X_{j}(q)\right), \quad 1 \leq p \neq q \leq d, \\
M_{k, n} & =\left(M_{k, n}(1), \ldots, M_{k, n}(d)\right), \\
M_{n} & =M_{0, n}, \\
M_{k, n}(p) & =\max _{k+1 \leq i \leq n} X_{i}(p), \\
M_{n}(p) & =M_{0, n}(p), \quad p=1, \ldots, d,
\end{aligned}
$$

and $u_{n}=\left(u_{n}(1), \ldots, u_{n}(d)\right)$ and $v_{n}=\left(v_{n}(1), \ldots, v_{n}(d)\right)$ are two real vectors. $u_{n}>v_{n}$ implies $u_{n}(p)>v_{n}(p)$ for all $p=1, \ldots$, $d$, and $a \ll b$ stands for $a=O(b)$. Finally, write $a_{n}=(2 \log n)^{1 / 2}$ and $b_{n}=a_{n}-(1 / 2) a_{n}^{-1} \log (4 \pi \log n)$. In this paper, the following results are obtained (proofs are given in Section 2).

\section{Results}

\subsection{Theorems}

Theorem 1. Let $\left\{X_{n}\right\}_{n=1}^{\infty}$ be a standardized normal sequence of d-dimensional random vector, satisfying $\left|r_{i j}(p)\right| \leq$ $\rho_{|j-i|}(p),\left|r_{i j}(p, q)\right| \leq \rho_{|j-i|}(p, q)$ for $i, j=1,2, \ldots$ and

$$
\begin{aligned}
& \sup _{\substack{n \geq 1 \\
1 \leq p \leq d}} \rho_{n}(p)<1, \\
& \sup _{n \geq 0} \quad \rho_{n}(p, q)<1,
\end{aligned}
$$

as $n \longrightarrow \infty$ and

$$
\begin{gathered}
\rho_{n}(p) \log n(\log \log n)^{(1+\varepsilon)}=O(1), \\
\rho_{n}(p, q) \log n(\log \log n)^{(1+\varepsilon)}=O(1) .
\end{gathered}
$$

Let the levels $\left\{u_{n i}(p)\right\}$ be such that $n\left(1-\Phi\left(\lambda_{n}(p)\right)\right.$ is bounded and $\lambda_{n}(p)=\min _{1 \leq i \leq n} u_{n i}(p) \geq c(\log n)^{1 / 2}$ for some $c>0$. If $\sum_{i=1}^{n}\left(1-\Phi\left(u_{n i}(p)\right)\right) \longrightarrow \tau_{p}$ for some $\tau_{p} \geq 0$ and $p=$ $1, \ldots, d$, then

$$
\lim _{n \longrightarrow \infty} \frac{1}{\log n} \sum_{k=1}^{n} \frac{1}{k} I\left(\bigcap_{i=1}^{k}\left(X_{i} \leq u_{k i}\right)\right)=\prod_{p=1}^{d} \exp \left(-\tau_{p}\right), \quad \text { a.s. }
$$

In particular, let $u_{n}(p)=a_{n}^{-1} x_{p}+b_{n}, x_{p} \in \mathbb{R}$ for $p=$ $1, \ldots, d$, and then

$$
\lim _{n \longrightarrow \infty} \frac{1}{\log n} \sum_{k=1}^{n} \frac{1}{k} I\left(M_{k} \leq u_{k}\right)=\prod_{p=1}^{d} \exp \left(-\exp \left(-x_{p}\right)\right) \text {, a.s. }
$$

Theorem 2. Let $\left\{X_{n}\right\}_{n=1}^{\infty}$ be a standardized normal $d$-dimensional random vector sequence satisfying

(a) $\delta=\max _{p \neq q}\left(\sup \left|r_{n}(p)\right|_{n \geq 1}, \sup _{n}(p, q)_{n \geq 0}\right)<1$

(b) there exists $\gamma \geq((2(1+\delta)) /(1-\delta))$, such that

$$
\begin{aligned}
& \frac{1}{n^{2}} \sum_{p=1}^{d} \sum_{1 \leq i<j \leq n}\left|r_{i j}(p)\right| \exp \left(\gamma\left|r_{i j}(p)\right| \log (j-i)\right) \\
& \ll(\log \log n)^{-(1+\varepsilon)}, \\
& \frac{1}{n^{2}} \sum_{1 \leq p \neq q \leq n}^{n} \sum_{1 \leq i \leq j \leq n}\left|r_{i j}(p, q)\right| \exp \left(\gamma\left|r_{i j}(p, q)\right| \log (j-i)\right) \\
& \ll(\log \log n)^{-(1+\varepsilon)},
\end{aligned}
$$

where $\varepsilon>0$. $d$, then

If $n\left(1-\Phi\left(u_{n}(p)\right)\right) \longrightarrow \tau_{p}, 0 \leq \tau_{p}<\infty$, for $p=1,2, \ldots$,

$$
\lim _{n \longrightarrow \infty} \frac{1}{\log n} \sum_{k=1}^{n} \frac{1}{k} I\left(M_{k} \leq U_{k}\right)=\prod_{p=1}^{d} \exp \left(-\tau_{p}\right), \quad \text { a.s. }
$$

Let $u_{n}(p)=a_{n}^{-1} x_{p}+b_{n}$, where $x_{p}$ are real numbers for $p=1, \ldots, d$, and then

$$
\lim _{n \longrightarrow \infty} \frac{1}{\log n} \sum_{k=1}^{n} \frac{1}{k} I\left(M_{k} \leq u_{k}\right)=\prod_{p=1}^{d} \exp \left(-\exp \left(-x_{p}\right)\right), \quad \text { a.s. }
$$

We shall be concerned with the maxima $M_{n}^{*}=\max _{1 \leq i \leq n} Y_{i}$ for a normal sequence $\left\{Y_{n}\right\}_{n=1}^{\infty}$ given by $Y_{n}=X_{n}+m_{n}$ where $\left\{X_{n}\right\}_{n=1}^{\infty}$ is a standardized normal sequence of $d$-dimensional vector with each component having a zero mean and unit standard deviation, and $\left\{m_{n}\right\}$ are added deterministic components. Define $m_{n}=\left(m_{n}(1), \ldots, m_{n}(d)\right)$, and we shall assume that the constant $m_{n}(p)$ is such that

$$
\begin{aligned}
\beta_{n}(p) & =\max _{1 \leq i \leq n}\left|m_{i}(p)\right|=o(\sqrt{\log n}), \\
p & =1, \ldots, d, \text { as } n \longrightarrow \infty
\end{aligned}
$$

Hence, the mean and the deviation of each component of $\left\{Y_{n}\right\}$ are $m_{n}(p)$ and 1 , and

$$
\begin{aligned}
\operatorname{Cov}\left(Y_{i}(p), Y_{j}(p)\right) & =r_{i j}(p), \\
\operatorname{Cov}\left(Y_{i}(p), Y_{j}(q)\right) & =r_{i j}(p, q), \\
1 & \leq p \neq q \leq d .
\end{aligned}
$$

Let $m_{n}^{*}=\left(m_{n}^{*}(1), \ldots, m_{n}^{*}(d)\right)$ be chosen such that $\left|m_{n}^{*}(p)\right| \leq \beta_{n}(p)$ and

$$
a_{n}\left(\max _{1 \leq i \leq n} m_{i}(p)-m_{i}^{*}(p)\right) \leq D, \quad p=1, \ldots, d,
$$

where $D$ is a constant.

Theorem 3. Let $\left\{Y_{n}\right\}_{n=1}^{\infty}$ be a normal sequence of $d$-dimensional vector defined above with $\left\{X_{n}\right\}_{n=1}^{\infty}$ satisfying the conditions of Theorem 1. Then,

$$
\begin{aligned}
& \lim _{n \longrightarrow \infty} \frac{1}{\log n} \sum_{k=1}^{n} \frac{1}{k} I\left(a_{k}\left(M_{k}^{*}-b_{k}^{*}-m_{k}^{*}\right) \leq x\right) \\
& \quad=\prod_{p=1}^{d} \exp \left(-e^{-x_{p}}\right), \quad \text { a.s. }
\end{aligned}
$$


where $M_{n}^{*}=\left(M_{n}^{*}(1), \ldots, M_{n}^{*}(d)\right)$ with $M_{n}^{*}(p)=$ $\max _{1 \leq i \leq n} Y_{i}(p)$ and $b_{n}^{*}=b_{n} I_{d}$ with $I_{d}=\underbrace{(1, \ldots, 1)}_{d}$ and $x=\left(x_{1}, \ldots, x_{d}\right)$.

2.2. Proofs of the Main Results. We need the following lemmas for the proofs of the main results.
Lemma 1. Let $\left\{\xi_{n}\right\}_{n=1}^{\infty}$ and $\left\{\eta_{n}\right\}_{n=1}^{\infty}$ be standardized normal sequences of $d$-dimensional random vector with $r_{i j}^{0}(p)=\operatorname{Cov}\left(\xi_{i}(p), \xi_{j}(p)\right), \quad r_{i j}^{0}(p, q)=\operatorname{Cov}\left(\xi_{i}(p), \xi_{j}(q)\right)$ and $\quad r_{i j}^{\prime}(p)=\operatorname{Cov}\left(\eta_{i}(p), \eta_{j}(p)\right), \quad r_{i j}^{\prime}(p, q)=\operatorname{Cov}\left(\eta_{i}(p)\right.$, $\left.\eta_{j}(q)\right)$. Let $\rho_{i j}(p)=\max \left(\left|r_{i j}^{0}(p)\right|,\left|r_{i j}^{\prime}(p)\right|\right), \quad \rho_{i j}(p, q)=$ $\max \left(\left|r_{i j}^{0}(p, q)\right|,\left|r_{i j}^{\prime}(p, q)\right|\right)$ and $u_{1}, \ldots, u_{n}$ be real vectors. Then,

$$
\begin{aligned}
& \left|P\left(\xi_{j} \leq u_{n j}, j=1, \ldots, n\right)-P\left(\eta_{j} \leq u_{n j}, j=1, \ldots, n\right)\right| \\
& \quad \leq K_{1} \sum_{p=1}^{d} \sum_{1 \leq i<j \leq n}\left|r_{i j}^{0}(p)-r_{i j}^{\prime}(p)\right|\left(1-\rho_{i j}^{2}(p)\right)^{-(1 / 2)} \exp \left(-\frac{u_{n i}^{2}(p)+u_{n j}^{2}(p)}{2\left(1+\rho_{i j}(p)\right)}\right) \\
& \quad+K_{2} \sum_{1 \leq p \neq q \leq d} \sum_{1 \leq i \leq j \leq n}\left|r_{i j}^{0}(p, q)-r_{i j}^{\prime}(p, q)\right|\left(1-\rho_{i j}^{2}(p, q)\right)^{-(1 / 2)} \exp \left(-\frac{u_{n i}^{2}(p)+u_{n j}^{2}(q)}{2\left(1+\rho_{i j}(p, q)\right)}\right),
\end{aligned}
$$

for constants $K_{1}, K_{2}$.

Proof. It follows from Theorem 4.2.1 in [22].

$$
\begin{aligned}
S_{n}:= & \sum_{1 \leq p \neq q \leq d} \sum_{1 \leq i \leq j \leq n}\left|r_{i j}(p, q)\right| \exp \left(-\frac{u_{n i}^{2}(p)+u_{n j}^{2}(q)}{2\left(1+\left|r_{i j}(p, q)\right|\right)}\right) \\
& \ll(\log \log n)^{-(1+\varepsilon)} .
\end{aligned}
$$

$d$-dimensional random vector satisfying equations (3) and

(4), and $n\left(1-\Phi\left(\lambda_{n}(p)\right)\right.$ be bounded for $p=1, \ldots, d$, then

$$
\begin{aligned}
S_{n}^{*}:= & \sum_{p=1}^{d} \sum_{1 \leq i<j \leq n}\left|r_{i j}(p)\right| \exp \left(-\frac{u_{n i}^{2}(p)+u_{n j}^{2}(p)}{2\left(1+\left|r_{i j}(p)\right|\right)}\right) \\
& \ll(\log \log n)^{-(1+\varepsilon)},
\end{aligned}
$$

Proof. By equation (3), we have $\sup _{1 \leq i \leq j \leq n, 1 \leq p \neq q \leq d} r_{i j}$ $(p, q)=\delta<1$. Define $\theta_{n}=e^{\eta\left(\lambda_{n}(p)\right)^{2}}$ for $\eta<\eta_{0}=((1-\delta) /$ $(2(1+\delta)))$. Split $S_{n}$ into two parts, then

$$
\begin{aligned}
S_{n}= & \sum_{1 \leq p \neq q \leq d} \sum_{\substack{j-i \leq \theta_{n} \\
1 \leq i \leq j \leq n}}\left|r_{i j}(p, q)\right| \exp \left(-\frac{u_{n i}^{2}(p)+u_{n j}^{2}(q)}{2\left(1+\left|r_{i j}(p, q)\right|\right)}\right) \\
& +\sum_{1 \leq p \neq q \leq d} \sum_{\substack{j-i>\theta_{n} \\
1 \leq i \leq j \leq n}}\left|r_{i j}(p, q)\right| \exp \left(-\frac{u_{n i}^{2}(p)+u_{n j}^{2}(q)}{2\left(1+\left|r_{i j}(p, q)\right|\right)}\right) \\
:= & S_{n}^{(1)}+S_{n}^{(2) .}
\end{aligned}
$$

Notice that $n\left(1-\Phi\left(\lambda_{n}(p)\right)\right)$ is bounded for $p=1, \ldots, d$, which implies that

$$
\begin{aligned}
\exp \left(-\frac{\lambda_{n}^{2}(p)}{2}\right) & \sim \frac{C \sqrt{2 \pi} \lambda_{n}(p)}{n}, \\
\lambda_{n}(p) & \sim \sqrt{2 \log n},
\end{aligned}
$$

for some constant $C>0, p=1, \ldots, d$, and then

$$
\begin{aligned}
S_{n}^{(1)} & \leq \sum_{1 \leq p \neq q \leq d} \delta \sum_{\substack{j-i \leq \theta_{n} \\
1 \leq i \leq j \leq n}} \exp \left(-\frac{u_{n i}^{2}(p)+u_{n j}^{2}(q)}{2(1+\delta)}\right) \\
& \leq \sum_{1 \leq p \neq q \leq d} \delta \theta_{n} n \exp \left(-\frac{\lambda_{n}^{2}(p)+\lambda_{n}^{2}(q)}{2(1+\delta)}\right) \\
& \ll \sum_{1 \leq p \neq q \leq d} n^{2\left(\eta-\eta_{0}\right)}(\log n)^{(1 /(1+\delta))-\eta} \\
& \ll(\log \log n)^{-(1+\varepsilon)},
\end{aligned}
$$


Now, consider $S_{n}^{(2)}$. Let $\delta_{t}=\sup _{j \geq t} \rho_{j}(p, q), 1 \leq p \neq q \leq d$ and $t=n^{\alpha}$. Since $n\left(1-\Phi\left(\lambda_{n}(p)\right)\right)$ is bounded as $n \longrightarrow \infty$ such that $\lambda_{n}^{2}(p)>c \log n$ for some $c>0$ and $\theta_{n}=$ $\exp \left(\eta \lambda_{n}^{2}(p)\right) \geq \exp \left(c^{2} \eta \log n\right)=n^{\alpha}, \alpha=c^{2} \eta>0$, and then

$$
\begin{aligned}
S_{n}^{(2)} & \leq \sum_{1 \leq p \neq q \leq d} \sum_{\substack{j-i>\theta_{n} \\
1 \leq i \leq j \leq n}} \delta_{t} \exp \left(-\frac{\lambda_{n}^{2}(p)+\lambda_{n}^{2}(q)}{2\left(1+\delta_{t}\right)}\right) \\
& \leq \sum_{1 \leq p \neq q \leq d} \delta_{t} n^{2} \exp \left(-\frac{\lambda_{n}^{2}(p)+\lambda_{n}^{2}(q)}{2\left(1+\delta_{t}\right)}\right) \\
& \ll \sum_{1 \leq p \neq q \leq d} \delta_{t}\left(n ( 1 - \Phi ( \lambda _ { n } ( p ) ) ) \left(n\left(1-\Phi\left(\lambda_{n}(q)\right)\right) \lambda_{n}(p) \exp \left(\frac{\lambda_{n}^{2}(p)}{2} \delta_{t}\right) \lambda_{n}(q) \exp \left(\frac{\lambda_{n}^{2}(q)}{2} \delta_{t}\right)\right.\right. \\
& \ll \sum_{1 \leq p \neq q \leq d} \delta_{t} \lambda_{n}(p) \exp \left(\frac{\lambda_{n}^{2}(p)}{2} \delta_{t}\right) \lambda_{n}(q) \exp \left(\frac{\lambda_{n}^{2}(q)}{2} \delta_{t}\right) \\
& \ll \sum_{1 \leq p \neq q \leq d} \sqrt{\delta_{t} \lambda_{n}^{2}(p)} \sqrt{\delta_{t} \lambda_{n}^{2}(q)} \exp \left(\frac{\lambda_{n}^{2}(p)}{2} \delta_{t}\right) \exp \left(\frac{\lambda_{n}^{2}(q)}{2} \delta_{t}\right) \\
& \ll \sum_{1 \leq p \neq q \leq d} \delta_{t} \log t \exp \left(\frac{1}{\alpha} \delta_{t} \log t\right) .
\end{aligned}
$$

As

$\delta_{t} \log t(\log \log t)^{-(1+\varepsilon)} \leq \sup _{j \geq t} \rho_{j}(p, q) \log j(\log \log j)^{-(1+\varepsilon)}=O(1)$,

we have

$$
\delta_{t} \log t \ll(\log \log n)^{-(1+\varepsilon)} \longrightarrow 0,
$$

and $\exp \left(\left(\lambda_{n}^{2}(p) / 2\right) \delta_{t}\right)$ is bounded. Then,

$$
S_{n}^{(2)} \ll(\log \log n)^{-(1+\varepsilon)} .
$$

Lemma 3. Let $\left\{X_{n}\right\}_{n=1}^{\infty}$ be a standardized normal $d$-dimensional random vector sequence satisfying the conditions (a) and (b) of Theorem 2, and then equations (15) and (16) hold.
Proof. The proof of Lemma 3 is similar to that of Lemma 2.

Lemma 4. Let $\left\{X_{n}\right\}_{n=1}^{\infty}$ be a standardized normal sequence of $d$-dimensional random vector satisfying Lemma 2 (Lemma 3), then

$$
\left|\operatorname{Cov}\left(I\left(\bigcap_{i=1}^{k}\left(X_{i} \leq u_{k i}\right)\right), I\left(\bigcap_{i=n+1}^{n}\left(X_{i} \leq u_{k i}\right)\right)\right)\right| \ll(\log \log n)^{-(1+\varepsilon)} .
$$

Proof. By Lemma 1,

$$
\begin{aligned}
\left|\operatorname{Cov}\left(I\left(\bigcap_{i=1}^{k}\left(X_{i} \leq u_{k i}\right)\right), I\left(\bigcap_{i=k+1}^{n}\left(X_{i} \leq u_{n i}\right)\right)\right)\right| \\
=\left|P\left(\left(\bigcap_{i=1}^{k}\left(X_{i} \leq u_{k i}\right)\right),\left(\bigcap_{i=k+1}^{n}\left(X_{i} \leq u_{n i}\right)\right)\right)-P\left(\bigcap_{i=1}^{k}\left(X_{i} \leq u_{k i}\right)\right) P\left(\bigcap_{i=k+1}^{n}\left(X_{i} \leq u_{n i}\right)\right)\right| \\
\leq \sum_{p=1}^{d} \sum_{i=1}^{k} \sum_{j=k+1}^{n}\left|r_{i j}(p)\right| \exp \left(-\frac{u_{k i}^{2}(p)+u_{n j}^{2}(p)}{2\left(1+\left|r_{i j}(p)\right|\right)}\right) \\
\quad+\sum_{1 \leq p \neq q \leq d} \sum_{i=1}^{k} \sum_{j=k+1}^{n}\left|r_{i j}(p, q)\right| \exp \left(-\frac{u_{k i}^{2}(p)+u_{n j}^{2}(q)}{2\left(1+\left|r_{i j}(p, q)\right|\right)}\right) \\
:=D_{n}+E_{n} .
\end{aligned}
$$


Both $D_{n}$ and $E_{n}$ are of order $(\log \log n)^{-(1+\varepsilon)}$, but we will only prove $E_{n} \ll(\log \log n)^{-(1+\varepsilon)}$. In fact,

$$
\begin{aligned}
E_{n} & =\sum_{1 \leq p \neq q \leq d} \sum_{i=1}^{k} \sum_{j=k+1}^{n}\left|r_{i j}(p, q)\right| \exp \left(-\frac{u_{k i}^{2}(p)+u_{n j}^{2}(q)}{2\left(1+\left|r_{i j}(p, q)\right|\right)}\right) \\
& \leq \sum_{1 \leq p \neq q \leq d} \sum_{\substack{1 \leq i \leq k \\
k+1 \leq j \leq n}}^{j-i<\theta_{n}}\left|r_{i j}(p, q)\right| \exp \left(-\frac{u_{k i}^{2}(p)+u_{n j}^{2}(q)}{2\left(1+\left|r_{i j}(p, q)\right|\right)}\right) \\
& +\sum_{1 \leq p \neq q \leq d} \sum_{\substack{1 \leq i \leq k \\
k+1 \leq j \leq n}}^{j-i<\theta_{n}}\left|r_{i j}(p, q)\right| \exp \left(-\frac{u_{k i}^{2}(p)+u_{n j}^{2}(q)}{2\left(1+\left|r_{i j}(p, q)\right|\right)}\right) \\
& :=E_{n}^{1}+E_{n}^{2} .
\end{aligned}
$$

We have

$$
\begin{aligned}
& E_{n}^{1} \leq \sum_{1 \leq p \neq q \leq d} \delta \sum_{1 \leq i \leq k k+1 \leq j \leq n}^{j-i<\theta_{n}} \exp \left(-\frac{\lambda_{k}^{2}(p)+\lambda_{n}^{2}(q)}{2(1+\delta)}\right) \\
& \leq \sum_{1 \leq p \neq q \leq d} \delta k \theta_{n} \exp \left(-\frac{\lambda_{k}^{2}(p)+\lambda_{n}^{2}(q)}{2(1+\delta)}\right) \\
& \leq \sum_{1 \leq p \neq q \leq d} \delta k \theta_{n}\left(\frac{\lambda_{k}(p)}{k}\right)^{1 /(1+\delta)}\left(\frac{\lambda_{n}(q)}{n}\right)^{1 /(1+\delta)} \\
& \leq \sum_{1 \leq p \neq q \leq d} \delta k^{1-(1 /(1+\delta))}(\log k)^{1 / 2(1+\delta)} n^{2 \eta-(1 /(1+\delta))}(\log n)^{(1 / 2(1+\delta))-\eta} \\
& \leq \sum_{1 \leq p \neq q \leq d} \delta n^{-2\left(\eta_{0}-\eta\right)}(\log n)^{(1 /(1+\delta))-\eta} \\
& \ll(\log \log n)^{-(1+\varepsilon)}, \\
& E_{n}^{2} \leq \sum_{1 \leq p \neq q \leq d} \sum_{1 \leq i \leq k+1 \leq j \leq n}^{j-i \geq \theta_{n}} \rho_{i j}(p, q) \exp \left(-\frac{u_{k i}^{2}(p)+u_{n j}^{2}(q)}{2\left(1+\rho_{i j}(p, q)\right)}\right) \\
& \leq \sum_{1 \leq p \neq q \leq d} \delta_{t} \sum_{i=1}^{k} \exp \left(-\frac{u_{k i}^{2}(p)}{2\left(1+\delta_{t}\right)}\right) \sum_{i=1}^{n} \exp \left(-\frac{u_{n i}^{2}(p)}{2\left(1+\delta_{t}\right)}\right) \\
& \leq \sum_{1 \leq p \neq q \leq d} \delta_{t} k \exp \left(-\frac{\lambda_{k}^{2}}{2}\right) \exp \left(\frac{1}{2} \lambda_{k}^{2} \delta_{t}\right) n \exp \left(-\frac{\lambda_{n}^{2}}{2}\right) \exp \left(\frac{1}{2} \lambda_{n}^{2} \delta_{t}\right) \\
& \ll \sum_{1 \leq p \neq q \leq d} \delta_{t} \log n \exp \left(\lambda_{n}^{2} \delta_{t}\right) \\
& \ll(\log \log n)^{-(1+\varepsilon)} .
\end{aligned}
$$


Remark 1. Under the conditions of Theorem 2, Lemma 4 can be proved by Lemma 1 and Lemma 3 .

Lemma 5. Let $\left\{X_{n}\right\}_{n=1}^{\infty}$ be a standardized normal sequence of d-dimensional random vector satisfying Lemma 2 (Lemma 3), then

$$
E\left|I\left(\bigcap_{i=1}^{n}\left(X_{i} \leq u_{n i}\right)\right)-I\left(\bigcap_{i=k+1}^{n}\left(X_{i} \leq u_{n i}\right)\right)\right| \ll \frac{k}{n}+(\log \log n)^{-(1+\varepsilon)},
$$

where $E$ denotes the mean.

Proof.

$$
\begin{aligned}
E \mid & \left|\left(\bigcap_{i=1}^{n}\left(X_{i} \leq u_{n i}\right)\right)-I\left(\bigcap_{i=k+1}^{n}\left(X_{i} \leq u_{n i}\right)\right)\right| \\
= & P\left(\bigcap_{i=1}^{n}\left(X_{i} \leq u_{n i}\right)\right)-P\left(\bigcap_{i=k+1}^{n}\left(X_{i} \leq u_{n i}\right)\right) \\
\leq & \left|P\left(\bigcap_{i=k+1}^{n}\left(X_{i} \leq u_{n i}\right)\right)-\prod_{p=1}^{d} \prod_{j=k+1}^{n} \Phi\left(u_{n j}(p)\right)\right| \\
& +\left|P\left(\bigcap_{i=1}^{n}\left(X_{i} \leq u_{n i}\right)\right)-\prod_{p=1}^{d} \prod_{j=1}^{n} \Phi\left(u_{n j}(p)\right)\right| \\
& +\left|\prod_{p=1}^{d} \prod_{j=k+1}^{n} \Phi\left(u_{n j}(p)\right)-\prod_{p=1}^{d} \prod_{j=1}^{n} \Phi\left(u_{n j}(p)\right)\right| \\
\triangleq & +B+C .
\end{aligned}
$$

By Lemma 1 and Lemma 2, we have

$$
\begin{aligned}
& A \ll(\log \log n)^{-(1+\varepsilon)}, \\
& B \ll(\log \log n)^{-(1+\varepsilon)} .
\end{aligned}
$$

Meanwhile,

$$
\begin{aligned}
& 0 \leq C=\prod_{j=k+1}^{n} \prod_{p=1}^{d} \Phi\left(u_{n j}(p)\right)-\prod_{j=1}^{n} \prod_{p=1}^{d} \Phi\left(u_{n j}(p)\right) \\
& \leq 1-\prod_{j=1}^{k} \prod_{p=1}^{d} \Phi\left(u_{n j}(p)\right) \\
& \leq \sum_{p=1}^{d} \sum_{j=1}^{k}\left(1-\Phi\left(u_{n j}(p)\right)\right) \\
& \leq \sum_{p=1}^{d} k\left(1-\Phi\left(\lambda_{n}(p)\right)\right) \\
& \leq \sum_{p=1}^{d} \frac{k}{n}\left[n\left(1-\Phi\left(\lambda_{n}(p)\right)\right)\right] \\
& \ll \frac{k}{n}
\end{aligned}
$$

from which the lemma follows.

Remark 2. Under the conditions of Theorem 2, Lemma 5 also can be proved using Lemma 1 and Lemma 3.
Lemma 6. Suppose that $\left\{X_{n}\right\}_{n=1}^{\infty}$ is a standardized normal sequence of d-dimensional random vector satisfying equation (3) of Theorem 1, and

$$
\begin{aligned}
\rho_{n}(p) \log n & \longrightarrow 0, \\
\rho_{n}(p, q) \log n & \longrightarrow 0 .
\end{aligned}
$$

As $\sum_{i=1}^{n}\left(1-\Phi\left(u_{n i}(p)\right)\right) \longrightarrow \tau_{p}$ for $\tau_{p} \geq 0$ and all

$$
\lim _{k \rightarrow \infty} P\left(\bigcap_{i=1}^{k}\left(X_{i} \leq u_{k i}\right)\right)=\prod_{p=1}^{d} \exp \left(-\tau_{p}\right) .
$$

Especially, let $u_{n}(p)=\left(1 / a_{n}\right) x_{p}+b_{n}$ with $x_{p} \in R$ for all $p=1, \ldots, d$; then,

$$
\lim _{k \longrightarrow \infty} P\left(M_{k} \leq u_{k}\right)=\prod_{p=1}^{d} \exp \left(-e^{-x_{p}}\right) .
$$

Proof. By Lemma 1, equation (32) can be obtained under the condition of equation (31), and the process of proof is similar to that of Lemma 2.

Lemma 7. Let $\left\{X_{n}\right\}_{n=1}^{\infty}$ be a standardized normal sequence of $d$-dimensional random vector satisfying (a) of Theorem 3 .

(c) There exists $\gamma \geq(2(1+\delta)) /(1-\delta)$, as $n \longrightarrow \infty$

$\frac{1}{n^{2}} \sum_{p=1}^{d} \sum_{1 \leq i<j \leq n}\left|r_{i j}(p)\right| \exp \left(\gamma\left|r_{i j}(p)\right| \log (j-i)\right) \longrightarrow 0$,

$\frac{1}{n^{2}} \sum_{1 \leq p \neq q \leq d} \sum_{1 \leq i \leq j \leq n}\left|r_{i j}(p, q)\right| \exp \left(\gamma\left|r_{i j}(p, q)\right| \log (j-i)\right) \longrightarrow 0$,

where $\varepsilon>0$. $d$, then

If $n\left(1-\Phi\left(u_{n}(p)\right)\right) \longrightarrow \tau_{p}, 0 \leq \tau_{p}<\infty$, for $p=1,2, \ldots$,

$$
\lim _{k \longrightarrow \infty} P\left(M_{k} \leq U_{k}\right)=\prod_{P=1}^{d} \exp \left(-\tau_{p}\right) .
$$

Let $u_{n}(p)=\left(1 / a_{n}\right) x_{p}+b_{n} \quad$ with $\quad x_{p} \in R \quad$ for all $p=1, \ldots, d$, then

$$
\lim _{k \longrightarrow \infty} P\left(M_{k} \leq u_{k}\right)=\prod_{p=1}^{d} \exp \left(-e^{-x_{p}}\right) .
$$

Proof. By Lemma 1, equation (36) can be obtained using equations (34) and (35), the method of proof is similar to that of Lemma 6

Lemma 8. Let $\left\{\Upsilon_{n}\right\}_{n=1}^{\infty}$ be a sequence of bounded random variables. If

$$
\operatorname{Var}\left(\sum_{k=1}^{n} \frac{1}{k} \Upsilon_{k}\right) \ll(\log n)^{2}(\log \log n)^{-(1+\varepsilon)}
$$


then

$$
\lim _{n \longrightarrow \infty} \frac{1}{\log n} \sum_{k=1}^{n} \frac{1}{k}\left(\Upsilon_{k}-E \Upsilon_{k}\right)=0, \quad \text { a.s. }
$$

Proof. See Lemma 3.1 in [11].

Proof of Theorem. 1. Let $\alpha_{k}=I\left(\cap_{i=1}^{n}\left(X_{i} \leq u_{k i}\right)\right)$, and

$$
\begin{aligned}
\operatorname{Var}\left(\sum_{k=1}^{n} \frac{1}{k} \alpha_{k}\right) & =\sum_{k=1}^{n} \frac{1}{k^{2}} \operatorname{Var}\left(\alpha_{k}\right)+2 \sum_{1 \leq k<l \leq n} \frac{\operatorname{Cov}\left(\alpha_{k}, \alpha_{l}\right)}{k l} \\
& \leq \sum_{k=1}^{n} \frac{1}{k^{2}}+2 \sum_{1 \leq k<l \leq n} \frac{\operatorname{Cov}\left(\alpha_{k}, \alpha_{l}\right)}{k l}
\end{aligned}
$$

By Lemma 4 and Lemma 5,

$$
\begin{aligned}
& \left|\operatorname{Cov}\left(\alpha_{k}, \alpha_{l}\right)\right|=\left|\operatorname{Cov}\left(I\left(\bigcap_{i=1}^{k}\left(X_{i} \leq u_{k i}\right)\right), I\left(\bigcap_{i=1}^{l}\left(X_{i} \leq u_{l i}\right)\right)\right)\right| \\
& \leq \mid \operatorname{Cov}\left(I\left(\bigcap_{i=1}^{k}\left(X_{i} \leq u_{k i}\right)\right)\right. \\
& \left.I\left(\bigcap_{i=1}^{l}\left(X_{i} \leq u_{l i}\right)\right)-I\left(\bigcap_{i=k+1}^{l}\left(X_{i} \leq u_{l i}\right)\right)\right) \\
& +\left|\operatorname{Cov}\left(I\left(\bigcap_{i=1}^{k}\left(X_{i} \leq u_{k i}\right)\right), I\left(\bigcap_{i=k+1}^{l}\left(X_{i} \leq u_{l i}\right)\right)\right)\right| \\
& \leq 2 E\left|I\left(\bigcap_{i=1}^{k}\left(X_{i} \leq u_{k i}\right)\right)-I\left(\bigcap_{i=k+1}^{l}\left(X_{i} \leq u_{l i}\right)\right)\right| \\
& \left.+\mid \operatorname{Cov}\left(I\left(\bigcap_{i=1}^{k}\left(X_{i} \leq u_{k i}\right)\right)\right), I\left(\bigcap_{i=k+1}^{l}\left(X_{i} \leq u_{l i}\right)\right)\right) \mid \\
& \ll \frac{k}{l}+(\log \log n)^{-(1+\varepsilon)}, \\
& \operatorname{Var}\left(\sum_{k=1}^{n} \frac{1}{k} \alpha_{k}\right) \leq \sum_{k=1}^{n} \frac{1}{k^{2}}+2 \sum_{1 \leq k<l \leq n} \frac{1}{k l}\left(\frac{k}{l}+(\log \log l)^{-(1+\varepsilon)}\right) \\
& =\sum_{k=1}^{n} \frac{1}{k^{2}}+2 \sum_{1 \leq k<l \leq n} \frac{1}{l^{2}}+2 \sum_{1 \leq k<l \leq n} \frac{1}{k l}(\log \log l)^{-(1+\varepsilon)} \\
& \ll \log n+2 \sum_{l=3}^{n} \frac{1}{l(\log \log l)^{(1+\varepsilon)}} \sum_{k=1}^{l-1} \frac{1}{k} \\
& \ll \log n+2 \sum_{l=3}^{n} \frac{\log l}{l(\log \log l)^{(1+\varepsilon)}} \\
& \ll(\log n)^{2}(\log \log n)^{-(1+\varepsilon)} .
\end{aligned}
$$

By Lemma 6 and Lemma 8, the proof of the theorem is completed.

Proof of Theorem. 2. Under the conditions of Theorem 2, using Lemma 4 and Lemma 5, we have

$$
\operatorname{Var}\left(\sum_{k=1}^{n} \frac{1}{k} \alpha_{k}\right) \ll(\log n)^{2}(\log \log n)^{-(1+\varepsilon)}
$$

By Lemma 7 and Lemma 8, Theorem 2 is obtained.

Proof of Theorem. 3. Write $u_{n i}(p)=u_{n}(p)+m_{n}^{*}(p)-m_{i}(p)$ where $u_{n}(p)=\left(x_{p} / a_{n}\right)+b_{n}, p=1, \ldots, d$.

$$
\begin{aligned}
P\left(a_{n}\left(M_{n}^{*}-b_{n}^{*}-m_{n}^{*}\right) \leq x\right) & =P\left(M_{n}^{*}=\frac{1}{a_{n}} x+b_{n}^{*}+m_{n}^{*}\right) \\
& =P\left(M_{n}^{*} \leq u_{n}+m_{n}^{*}\right) \\
& =P\left(M_{n} \leq u_{n i}\right) .
\end{aligned}
$$

By Theorem 1, it is needed to check that $n\left(1-\Phi\left(\lambda_{n}(p)\right)\right)$ is bounded with $\lambda_{n}(p)=\min _{1 \leq i \leq n} u_{n i}(p)=u_{n}(p)+$ $m_{n}^{*}(p)-\max _{1 \leq i \leq n} m_{i}(p)$. Notice that $\beta_{n}(p)=$ $\max _{1 \leq i \leq n}\left|m_{i}(p)\right|=o(\sqrt{\log n})$ as $n \longrightarrow \infty,\left|m_{n}^{*}(p)\right| \leq \beta_{n}(p)$ and $a_{n}\left(\max _{1 \leq i \leq n} m_{i}(p)-m_{i}^{*}(p)\right) \leq D, p=1, \ldots, d$, and

$$
\begin{aligned}
u_{n}(p)\left(\max _{1 \leq i \leq n} m_{i}(p)-m_{n}^{*}(p)\right)-\frac{1}{2}\left(\max _{1 \leq i \leq n} m_{i}(p)-m_{n}^{*}(p)\right)^{2} \\
=a_{n}\left(\max _{1 \leq i \leq n} m_{i}(p)-m_{n}^{*}(p)\right) \\
\quad\left(1-\frac{\log (4 \pi \log n)}{a_{n}}+\frac{x_{p}}{a_{n}^{2}}-\frac{\max _{1 \leq i \leq n} m_{i}(p)-m_{n}^{*}(p)}{2 a_{n}}\right) \\
\leq D(1+o(1)),
\end{aligned}
$$

for large $n$. Hence,

$$
\begin{aligned}
n(1 & \left.-\Phi\left(\lambda_{n}(p)\right)\right) \\
& \sim n \lambda_{n}^{-1}(p) \exp \left(-\frac{1}{2}\left(u_{n}(p)+m_{n}^{*}(p)-\max _{1 \leq i \leq n} m_{i}(p)\right)^{2}\right) \\
& \sim n\left(1-\Phi\left(u_{n}(p)\right)\right) \exp \left(-u_{n}(p)\left(m_{n}^{*}(p)-\max _{1 \leq i \leq n} m_{i}(p)\right)\right. \\
& \left.\quad-\frac{1}{2}\left(m_{n}^{*}(p)-\max _{1 \leq i \leq n} m_{i}(p)\right)^{2}\right) \\
\leq & D^{\prime} .
\end{aligned}
$$

$n\left(1-\Phi\left(\lambda_{n}(p)\right)\right)$ is bounded and satisfies the conditions of Theorem 1 . As $u_{n}(p)=\left(x_{p} / a_{n}\right)+b_{n}$, Theorem 3 can be proved. 
TABLE 1: The rate of convergence.

\begin{tabular}{lccccccc}
\hline$N$ & $10^{3}$ & $10^{4}$ & $10^{5}$ & $10^{6}$ & $10^{7}$ & $10^{8}$ & $10^{9}$ \\
\hline Value & 1.0836 & 1.0627 & 1.0501 & 1.0408 & 1.0358 & 1.0318 & 1.0279 \\
\hline
\end{tabular}

${ }^{\mathrm{a}} \mathrm{N}$ denotes the number of random variables.

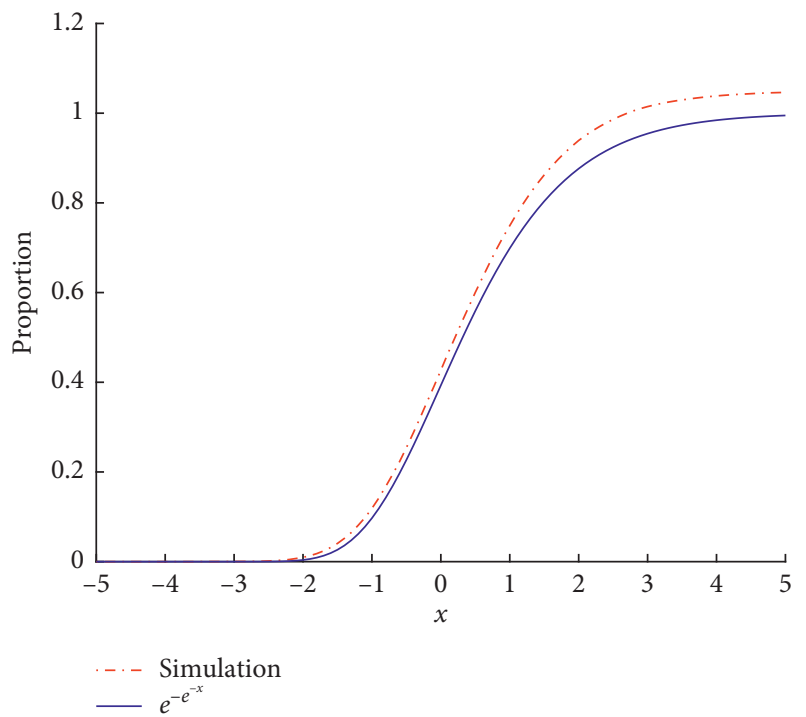

Figure 1: Simulating almost sure central limit theorem for onedimensional random variable sequence.

2.3. Simulation. The almost sure central limit theorems for the maximum of the multivariate normal sequences are complete. Simulating the almost sure convergence for the maxima, which visually describe the theorems, is not performed in previous papers $[12,13,15,16,23-26]$. To construct a suitable standardized nonstationary normal sequence is the key to the simulation.

Let $\left\{Z_{1}, Z_{2}, \ldots\right\}$ be an independent random sequence from the standardized normal distribution $N(0,1)$. Set $X_{i}=(1 / \sqrt{i}) Z_{1}+((\sqrt{i-1}) / \sqrt{i}) Z_{i}$, then $E\left(X_{i}\right)=0$ and $\operatorname{Var}\left(X_{i}\right)=1$, that is, $X_{i} \sim N(0,1), i=1,2, \ldots$ As the correlation coefficient of $X_{i}$ and $X_{j}, r_{i j}=\operatorname{corr}\left(X_{i}, X_{j}\right)=$ $(1 / \sqrt{i j}) \stackrel{j=i+n}{=}(1 /(\sqrt{i(n+i)})),\left\{X_{1}, X_{2}, \ldots\right\}$ be a standardized nonstationary normal random variable sequence. For $r_{i j} \leq(1 / \sqrt{n})$, the sequence satisfies equations (3) and (4). When $\left\{X_{1}, X_{2}, \ldots\right\}$ are one-dimensional variables and $\lambda_{n}=a_{n}^{-1} x+b_{n}$, equation (6) of Theorem 2 holds.

Theorem 2 is simulated based on the standardized nonstationary normal random variable sequence $\left\{X_{1}, X_{2}, \ldots\right\}$ by Matlab. $N$ denotes the number of random variable sequence $\left\{X_{1}, X_{2}, \ldots\right\}$ and $M_{k}=\max _{1 \leq i \leq k} X_{i}$. $a_{n}=(2 \log n)^{(1 / 2)}, \quad b_{n}=a_{n}-(1 / 2) a_{n}^{-1} \log (4 \pi \log n)$, especially $a_{1}=1, b_{1}=0$ and $\lambda_{n}=a_{n}^{-1} x+b_{n}$. We note that $(1 /(\log n)) \sum_{k=1}^{n}(1 / k)$ is greater than 1 and converges to 1 , but the rate of convergence is slow. The value $(1 /(\log n)) \sum_{k=1}^{n} 1 / k$ and the number of the random variables $N$ are displayed in Table 1 . The fitting effect gets better with the number of random variable $N$ increasing. The detailed simulation is shown in Figure 1.

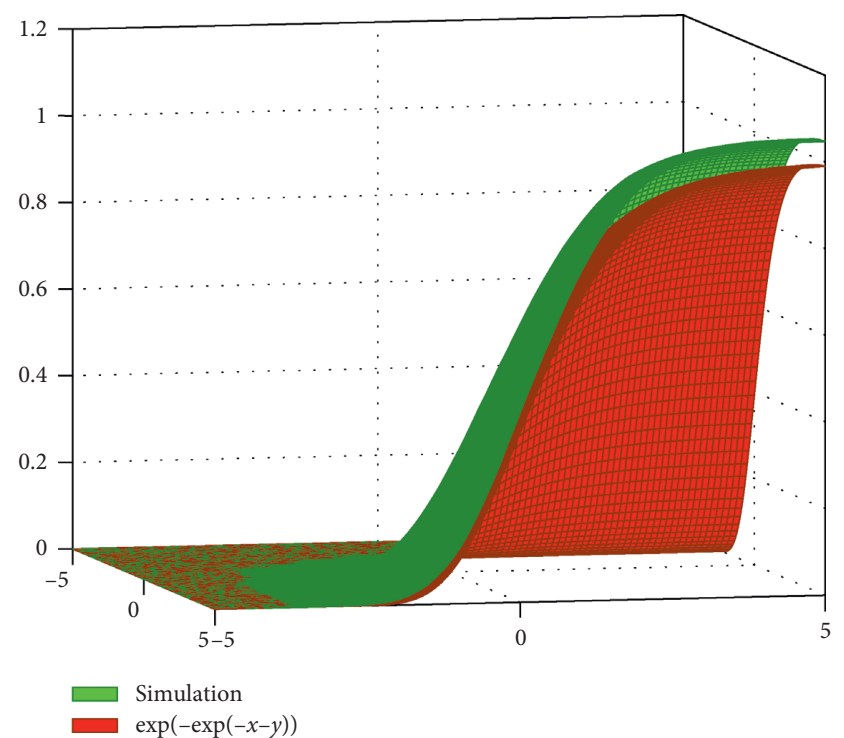

Figure 2: Simulating almost sure central limit theorem for twodimensional random variable sequence.

Let $Y_{i}=-(1 / \sqrt{i}) Z_{1}+((\sqrt{i-1}) / \sqrt{i}) Z_{i}$, then $E\left(Y_{i}\right)=0$, and $\operatorname{Var}\left(Y_{i}\right)=1, Y_{i} \sim N(0,1), i=1,2, \ldots \xi_{i}=\left(X_{i}, Y_{i}\right)$ is a two-dimensional random vector.

$$
\begin{gathered}
r_{i j}(2)=\operatorname{corr}\left(Y_{i}, Y_{j}\right)=\frac{1}{\sqrt{i j}}(i \neq j), \\
r_{i j}(1,2)=\operatorname{corr}\left(X_{i}, Y_{j}\right)= \begin{cases}\frac{1}{\sqrt{i j}}, & i \neq j, \\
\frac{i-2}{i}, & i=j .\end{cases}
\end{gathered}
$$

$\left\{\xi_{1}, \xi_{2}, \ldots\right\}$ is a standardized nonstationary normal sequence of two-dimensional random vector satisfying equations (3) and (4), and then Theorem 2 holds as $\left\{\xi_{1}, \xi_{2}, \ldots\right\}$ is a 2 -dimensional random vector. The detailed simulation is shown in Figure 2.

\section{Conclusions}

The extreme value theory deals with extreme phenomena which are less likely to occur, but more harmful [1-3]. Almost sure limit theorems, nice behavior of convergence, for the maxima of normal vector sequences are put forward and proved. Figures 1 and 2 display good simulation for two examples and show the rates of the convergence visually, which promote the intuitive understanding about the almost sure central limit theorems for the maximum of nonstationary normal sequences of random vectors.

\section{Data Availability}

All data generated or analyzed during this study are included within this manuscript. If necessary, other relevant data can be made available from the corresponding author via mail upon request. 


\section{Conflicts of Interest}

The authors declare that there are no conflicts of interest regarding the publication of this article.

\section{Acknowledgments}

This work was supported by the National Natural Science Foundation of China (61374183 and 51535005), the Research Fund of State Key Laboratory of Mechanics and Control of Mechanical Structures (MCMS-I-0418K01 and MCMSI-0418Y01), the Fundamental Research Funds for the Central Universities (NC2018001, NP2019301, and NJ2019002), a Project Funded by the Priority Academic Program Development of Jiangsu Higher Education Institutions, and the Higher Education Institution Key Research Project Plan of Henan Province, China (20B110005).

\section{References}

[1] J. Galambos, "The asymptotic theory of extreme order statistics," Journal of the American Statistical Association, vol. 75, no. 370, 1980.

[2] B. Jan, Y. Goegebeur, J. Segers, and J. L. Teugels, Statistics of Extremes: Theory and Applications, John Wiley \& Sons, Hoboken, NJ, USA, 2006.

[3] S. I. Resnick, Extreme Values, Regular Variation and Point Processes, Springer, Berlin, Germany, 2013.

[4] P. Eduardo Oliveira, Almost Sure Convergence, Springer, Berlin, Germany, 2012.

[5] S. Cheng, P. Liang, and Y. Qi, "Almost sure convergence in extreme value theory," Mathematische Nachrichten, vol. 190, no. 1, pp. 43-50, 1998.

[6] G. A. Brosamler, "An almost everywhere central limit theorem," in Mathematical Proceedings of the Cambridge Philosophical Society, vol. 104, pp. 561-574, Cambridge University Press, Cambridge, UK, 1988.

[7] J. Hüsler and M. Schüpbach, "Limit results for maxima in non-stationary multivariate Gaussian sequences," Stochastic Processes and Their Applications, vol. 28, no. 1, pp. 91-99, 1988.

[8] M. T. Lacey and W. Philipp, "A note on the almost sure central limit theorem," Statistics \& Probability Letters, vol. 9, no. 3, pp. 201-205, 1990.

[9] I. Fahrner and U. Stadtmüller, "On almost sure max-limit theorems," Statistics \& Probability Letters, vol. 37, no. 3, pp. 229-236, 1998.

[10] I. Berkes, E. Csáki, and L. Horváth, "Almost sure central limit theorems under minimal conditions," Statistics \& Probability Letters, vol. 37, no. 1, pp. 67-76, 1998.

[11] E. Csáki and K. Gonchigdanzan, "Almost sure limit theorems for the maximum of stationary Gaussian sequences," Statistics \& Probability Letters, vol. 58, no. 2, pp. 195-203, 2002.

[12] S. Chen and Z. Lin, "Almost sure max-limits for nonstationary Gaussian sequence," Statistics \& Probability Letters, vol. 76, no. 11, pp. 1175-1184, 2006.

[13] Z. Chen, Z. Peng, and H. Zhang, "An almost sure limit theorem for the maxima of multivariate stationary Gaussian sequences," Journal of the Australian Mathematical Society, vol. 86, no. 3, pp. 315-321, 2009.

[14] J. Hüsler, "Multivariate extreme values in stationary random sequences," Stochastic Processes and Their Applications, vol. 35, no. 1, pp. 99-108, 1990.
[15] B. Tong and Z. X. Peng, "On almost sure max-limit theorems of complete and incomplete samples from stationary sequences," Acta Mathematica Sinica, English Series, vol. 27, no. 7, pp. 1323-1332, 2011.

[16] Z. Peng, Z. Weng, and S. Nadarajah, "Almost sure limit theorems of extremes of complete and incomplete samples of stationary sequences," Extremes, vol. 13, no. 4, pp. 463-480, 2010.

[17] Q.-p. Zang, Z.-x. Wang, and K.-a. Fu, "A note on almost sure central limit theorem in the joint version for the maxima and sums," Journal of Inequalities and Applications, vol. 2010, no. 1, Article ID 234964, 2010.

[18] Z. Tan and Y. Wang, "Almost sure central limit theorem for the maxima and sums of stationary Gaussian sequences," Journal of the Korean Statistical Society, vol. 40, no. 3, pp. 347-355, 2011.

[19] Z. Panga and L. Pereira, "On the almost sure convergence for the joint version of maxima and minima of stationary sequences," Statistics \& Probability Letters, vol. 154, p. 108540, 2019.

[20] Z. Weng, Z. Peng, and S. Nadarajah, "The almost sure limit theorem for the maxima and minima of strongly dependent Gaussian vector sequences," Extremes, vol. 15, no. 3, pp. 389-406, 2012.

[21] X. Zeng and Q. Wu, "The improved results in almost sure central limit theorem for the maxima of strongly dependent stationary Gaussian vector sequences," Journal of Inequalities and Applications, vol. 2015, no. 1, p. 224, 2015.

[22] M. R. Leadbetter, G. Lindgren, and H. Rootzén, Extremes and Related Properties of Random Sequences and Processes, Springer Science \& Business Media, Berlin, Germany, 2012.

[23] S. Chen and Z. Lin, "Almost sure functional central limit theorems for weakly dependent sequences," Statistics \& Probability Letters, vol. 78, no. 13, pp. 1683-1693, 2008.

[24] C. Shouquan and L. Zhengyan, "Almost sure limit theorems for a stationary normal sequence," Applied Mathematics Letters, vol. 20, no. 3, pp. 316-322, 2007.

[25] F. Lin, Y. Fu, and Y. Jiang, "Almost sure limit theorems for the maxima of some strongly dependent Gaussian sequences," Computers \& Mathematics with Applications, vol. 62, no. 2, pp. 635-640, 2011.

[26] Z. Tan and Z. Peng, "Almost sure convergence for non-stationary random sequences," Statistics \& Probability Letters, vol. 79, no. 7, pp. 857-863, 2009. 